\title{
Comorbilidades, calidad de sueño y calidad de vida en pacientes con cáncer pulmonar localmente avanzado
}

\author{
Luis Torre-Bouscoulet, * Oscar Arrieta-Rodríguez, ${ }^{,}$Rogelio Pérez-Padilla, * Abigail Guzmán-Barragán,* \\ Margarita Reyes-Zúñiga, * David Martínez-Briseño,* Rosario Fernández-Plata,* \\ Cecilia García-Sancho* $\bowtie$
}

\author{
*Instituto Nacional de Enfermedades Respiratorias Ismael Cosío Villegas, México, D.F.; "Instituto Nacional de Cancerología, \\ México, D.F. \\ Trabajo recibido: 07-V-2015; aceptado: 19-V-2015
}

\begin{abstract}
RESUMEN. Antecedentes: Los pacientes con cáncer pulmonar presentan condiciones médicas y comorbilidades que disminuyen la calidad de sueño y la calidad de vida y que pueden afectar la respuesta al tratamiento oncológico y el pronóstico. Objetivo: El propósito de este estudio es investigar, en pacientes con cáncer pulmonar localmente avanzado, la prevalencia de condiciones médicas y comorbilidades que podrían ser revertidas desde antes del tratamiento oncológico. Material y métodos: Diseño del estudio: evaluación inicial de pacientes consecutivos con diagnóstico de cáncer pulmonar de células no pequeñas etapas IIIA y IIIB. Para investigar las comorbilidades se utilizó el cuestionario PLATINO (www.platino-alat.org/docs/cuestionario_platino_ mexico.pdf). Se aplicaron la escala de disnea (MRC), el índice de calidad del sueño de Pittsburg (PSQI) y la escala de calidad de vida de EuroQL. Las pruebas de función pulmonar fueron: espirometría, capacidad de difusión pulmonar de monóxido de carbono (DLCO) y gasometría arterial (ATS/ERS 2005). Las escalas de PSQI y EuroQL se analizaron de acuerdo a los criterios de los autores. Resultados: Se reclutaron 46 pacientes; a) $32.6 \%$ de los pacientes refirieron tener una sola comorbilidad y $13.1 \%$ refirieron disnea; b) la prevalencia de EPOC fue de $39.1 \%$ y $16.7 \%$ mostraron hipoxemia; c) $50 \%$ reportaron una pobre calidad de sueño; d) el promedio del puntaje de calidad de vida fue alto $(0.848$ [EE \pm 0.185$]$ ); $45.7 \%$ refirieron dolor y $19.6 \%$ síntomas de ansiedad y depresión; e) la calidad del sueño se asoció significativamente con dolor y síntomas de ansiedad y depresión. Conclusión: Existen comorbilidades y condiciones médicas en pacientes con cáncer pulmonar localmente avanzado que podrían ser tratadas antes de iniciar el tratamiento con radio y quimioterapia para mejorar el diagnóstico de estos pacientes.
\end{abstract}

Palabras clave: Cáncer pulmonar, calidad de vida, función pulmonar, sueño.

ABSTRACT. Background: Patients with advanced lung cancer show medical conditions and comorbidities which decreases the sleep quality and quality of life that could affect the oncological treatment response and the prognosis of patients. Objective: The proposal of this study was to investigate, in patients with advanced lung cancer, the prevalence of medical conditions and comorbidities that could be reverted before radiotherapy or chemotherapy treatment. Material and methods: Study design: baseline assessment of consecutive patients with diagnosed of non-small lung cancer stages III-A and III-B. To investigate the comorbidities we use PLATINO questionnaire (www.platino-alat.org/docs/ cuestionario_platino_mexico.pdf). We applied also dyspnea scale MRC, the Pittsburgh sleep quality index (PSQI) and EuroQL. Lung function tests were spirometry, carbon monoxide diffusing capacity (DLCO) and gasometry (ATS/ERS 2005). PSQI and EuroQL scales were analyzed according authors criteria. Results: 46 patients were recruited; a) $32.6 \%$ of patients have a comorbid condition and $13.1 \%$ dyspnea; b) COPD prevalence was of $39.1 \%$ and $16.7 \%$ show hypoxemia; c) $50 \%$ reported a poor quality of sleep; d) the mean of quality of life score was high (0.848 [SE \pm 0.185$]$ ]); $45.7 \%$ refer pain and $19.6 \%$ anxiety and depression symptoms; e) sleep quality was significantly associated with pain and anxiety and depression symptoms. Conclusions: There are comorbidities and medical conditions in advanced lung cancer patients that could be treated before the radio and chemotherapy to improve the prognosis.

Key words: Lung cancer, lung function, quality of life, sleep.

\section{INTRODUCCIÓN}

Los pacientes con cáncer pulmonar presentan frecuentemente condiciones médicas independientes que podrían disminuir la calidad de vida y la calidad de sueño, ya sea por la presencia de enfermedades relacionadas con la edad o con el tabaquismo, o bien con otros factores de riesgo. Además, las comorbilidades podrían afectar la respuesta al tratamiento oncológico con consecuencias sobre el pronóstico. Para algunas de esas comorbilidades existen tratamientos eficaces que deberían iniciarse antes de quimio o radioterapia. Es posible que la identificación y tratamiento de las condiciones médicas subyacentes en pacientes con 
cáncer pulmonar localmente avanzado constituya una estrategia útil para mejorar el pronóstico. ${ }^{1}$ El propósito de este estudio es investigar, en pacientes con cáncer pulmonar localmente avanzado, la prevalencia de condiciones médicas que podrían ser revertidas o controladas antes del tratamiento oncológico. ${ }^{2}$ La hipótesis del estudio es que los pacientes con cáncer pulmonar localmente avanzado cursan con elevada frecuencia de comorbilidades potencialmente modificables antes de ser sometidos a quimioterapia y radioterapia.

\section{MATERIAL Y MÉTODOS}

Este reporte es la evaluación inicial de pacientes con cáncer pulmonar localmente avanzado. La totalidad de los pacientes fueron referidos de la Clínica de Cáncer Pulmonar del Instituto Nacional de Cancerología al Departamento de Fisiología Respiratoria del Instituto Nacional de Enfermedades Respiratorias Ismael Cosío Villegas. Ambos son institutos de referencia nacional de tercer nivel dedicados principalmente a la docencia e investigación; atienden primordialmente pacientes sin seguro de zonas cercanas a la institución.

Se incluyeron de forma consecutiva a pacientes con diagnóstico incidente de cáncer pulmonar de células no pequeñas (NSCLC non-small cell lung cancer, por sus siglas en inglés), localmente avanzado (etapas IIIA y IIIB), con confirmación histológica, vírgenes de tratamiento con quimio y radioterapia, que fueran candidatos a tratamiento con radioterapia y que consintieran en participar en el proyecto. La inclusión de pacientes se llevó a cabo en un período comprendido de 2012 a 2015. El protocolo y carta de consentimiento informado fueron aprobados por los comités institucionales (INER C12-12) y el estudio fue registrado en ClinicalTrials.gov: NCT01580579.

Las comorbilidades fueron investigadas mediante un cuestionario estandarizado utilizado previamente en el estudio epidemiológico PLATINO. PLATINO es una encuesta de base poblacional llevada a cabo en cinco ciudades de Latinoamérica con el objetivo principal de investigar la prevalencia de enfermedad pulmonar obstructiva crónica (EPOC). La metodología de este estudio se ha descrito previamente ${ }^{3}$ y los resultados de ese estudio ya han sido publicados. ${ }^{4}$ El cuestionario PLATINO está disponible en la página Web de PLATINO (www. platino-alat.org/docs/cuestionario_platino_mexico.pdf). Se aplicó la escala de disnea MRC (Medical Research Council) que tiene un puntaje de 0 a 4; 0 significa no disnea y 4 es disnea con actividades mínimas o disnea en reposo. El puntaje va de (0) ausencia de disnea excepto al realizar ejercicio intenso; (1) disnea al caminar de prisa en llano, o al caminar subiendo una pendiente poco pronunciada; (2) la disnea le produce una incapacidad de mantener el paso de otras personas de la misma edad caminando en llano o tener que parar a descansar al andar en llano al propio paso; (3) la disnea hace que tenga que parar a descansar al caminar unos 100 metros o después de pocos minutos de caminar en llano; (4) la disnea impide al paciente salir de casa o aparece con actividades como vestirse o desvestirse. ${ }^{5}$ Para el análisis se reagruparon a los pacientes con disnea grados 1 y 2 y con disnea grados 3 y 4 .

La calidad de sueño se exploró mediante el índice de calidad del sueño de Pittsburg (Pittsburgh Sleep Quality Index [PSQI]). ${ }^{6}$ EI PSQI explora siete condiciones relacionadas al sueño: duración, alteraciones durante el sueño, latencia al sueño, días de disfunción por somnolencia diurna, eficiencia del sueño, calidad del sueño y necesidad de medicamentos para dormir. Para cada condición existe un puntaje que va de 0 a 3 , siendo el 0 el mejor y 3 el peor puntaje. El puntaje total de calidad de sueño se clasifica como $>5$ : mala calidad del sueño, $\leq 5$ buena calidad del sueño.

La escala de calidad de vida fue utilizada fue EuroQL. Esta escala está conformada por 5 dominios: 1) movilidad; 2) cuidado personal; 3) actividades cotidianas; 4) malestar y dolor y 5) ansiedad y depresión. Cada uno de estos dominios se puede reportar como: (1) sin problemas; (2) algunos problemas; y (3) problemas graves. El puntaje total de la evaluación de la escala va de un valor de 0.001 (error estándar 0.022) a 1.0 (error estándar 0.0), en el cual el peor puntaje es el cercano a 0 y el mejor puntaje es el cercano a 1.0.7

Las pruebas de función respiratoria que se llevaron a cabo incluyeron: espirometría, difusión pulmonar de monóxido de carbono (DLCO) y gasometría arterial. La espirometría y la DLCO se llevaron a cabo siguiendo los estándares ATS/ERS 2005 con un equipo NDD EasyOne Pro (NDD Switzerland). ${ }^{8}$ Después de realizar la prueba de Allen para verificar la permeabilidad vascular, se obtuvo una muestra de sangre mediante punción de la arteria radial de la mano no dominante. La muestra se procesó en un analizador de gases Radiometer Copenhague. Antes de realizar las pruebas de función respiratoria, se obtuvo el peso con una báscula electrónica (escala solar HS-301 Tanita Corporation, Inc., USA); la estatura con un estadímetro (208 Seca Corporation, USA) en dos ocasiones y se utilizó el promedio de los dos valores para el análisis.

\section{Análisis estadístico}

Para la descripción de los datos utilizamos medias y desviaciones estándar o frecuencias y porcentajes según fuera el caso. Para comparar variables categóricas 
se utilizó la prueba de $\chi^{2}$ y para las variables continuas la prueba de rangos de Wilcoxon. Se realizó un análisis univariado para explorar cofactores asociados a pobre calidad de sueño y a menor calidad de vida. EI PSQI y EuroQL fueron calificados de acuerdo a lo señalado por sus autores. Las variables independientes que resultaron asociadas con un valor de $p<0.1$ fueron incluidas en modelos multivariados. La fuerza de las asociaciones se expresan en razones de momios (RM) e intervalos de confianza al 95\% (IC95\%). Se consideró como significativo a un valor de $p<0.05$. El análisis estadístico se realizó con el programa estadístico STATA versión 9.0.9

\section{RESULTADOS}

Se incluyeron en el estudio 46 pacientes con diagnóstico confirmado de cáncer pulmonar localmente avanzado; de ellos, 24 (52.2\%) fueron hombres, con un promedio de edad de $62(\mathrm{DE} \pm 14)$ años. Más de la mitad habían fumado alguna vez en su vida más de 100 cigarros (56.5\%). Sólo un paciente continuaba fumando al momento del ingreso al estudio (tabla 1).

En la tabla 2 se presentan las comorbilidades diagnosticadas por el médico o referidas por los pacientes. Las comorbilidades que se reportaron más frecuentemente fueron: gastritis y úlceras (37\%); hipertensión arterial sistémica $(28.3 \%)$, diabetes mellitus tipo 2 (17.4\%) y obesidad (15.6\%). Del total de participantes $32.6 \%$ presentó una sola comorbilidad. El promedio de comorbilidades para el grupo fue de 1.02 (DE \pm 0.99 ). En la tabla 3 se muestran los resultados de la espirometría, DLCO y gasometría. La prevalencia de EPOC definida como el cociente volumen espiratorio forzado en el primer segundo/capacidad vital forzada $\left(\mathrm{VEF}_{1} /\right.$ CVF) crudo < 0.7 fue de 18/46 (39.1\%). La DLCO promedio fue de $18.2 \pm 7.3 \mathrm{~mL} / \mathrm{min} / \mathrm{mmHg}$ y el porcentaje del predicho de la DLCO fue de $78.8 \pm 27 \%$. La presión parcial de oxígeno en sangre arterial $\left(\mathrm{PaO}_{2}\right)$ fue de 62.7 $(\mathrm{DE} \pm 7.1) \mathrm{mmHg}$. El $16.7 \%$ de los pacientes presentaban hipoxemia clara y $14.3 \%$ limítrofe. Es decir, el $31 \%$ de estos pacientes cumplían el criterio estándar para prescripción de oxígeno suplementario.

Tabla 1. Características generales de los pacientes con cáncer pulmonar localmente avanzado (etapas IIIA y IIIB).

\begin{tabular}{lc}
\hline Característica & Valor \\
\hline Hombres, $\mathrm{n}(\%)$ & $24(52.2)$ \\
Edad (años) [media $\pm \mathrm{DE}]$ & $62 \pm 14$ \\
Escolaridad en años [media $\pm \mathrm{DE}]$ & $11.1 \pm 7.4$ \\
Escolaridad formal $\leq 6$ años, $\mathrm{n}(\%)$ & $6(13.0)$ \\
Alguna vez fumó más de 100 cigarros, $\mathrm{n}(\%)$ & $26(56.5)$ \\
\hline
\end{tabular}

En relación a la calidad del sueño, el puntaje total del PSQI fue de $6.2 \pm 4.1$, con una mediana de 5.5 (mínimo 1 , máximo 19$)$. Identificamos $23 / 46$ pacientes $(50 \%)$ con mala calidad del sueño (puntaje total > 5) y 23/46 (50\%) con buena calidad del sueño (puntaje total $\leq 5$ ). El $67.4 \%$ de los pacientes refirieron dormir $<7$ horas, en tanto $8 / 46(17.7 \%)$ manifestaron insomnio. En el dominio de dolor del PSQI, 19 pacientes (41.3\%) refirieron haber padecido dolor durante el sueño (tabla 4).

En cuanto a la calidad de vida del total de pacientes, 21 refirieron tener algunos o muchos problemas de malestar y dolor (45.7\%) y 9 reportaron algunos o muchos problemas de ansiedad y depresión (19.6\%). El puntaje

Tabla 2. Estados comórbidos de los pacientes con cáncer pulmonar localmente avanzado (etapas IIIA y IIIB) $(n=46)$.

\begin{tabular}{lc}
\hline Característica & Valor \\
\hline Enfermedad del corazón, $\mathrm{n}(\%)$ & $6(13.0)$ \\
Hipertensión arterial sistémica, $\mathrm{n}(\%)$ & $13(28.3)$ \\
Embolia, isquemia o derrame cerebral, $\mathrm{n}(\%)$ & $2(4.4)$ \\
Diabetes mellitus, $\mathrm{n}(\%)$ & $8(17.4)$ \\
Tuberculosis pulmonar, $\mathrm{n}(\%)$ & $1(2.2)$ \\
Gastritis o úlcera, $\mathrm{n}(\%)$ & $17(37)$ \\
$\mathrm{IMC}\left(\mathrm{k} / \mathrm{m}^{2}\right)[\mathrm{media} \pm \mathrm{DE}]$ & $25.8 \pm 4.8$ \\
Bajo peso $\left(\mathrm{IMC}<18.5\left[\mathrm{k} / \mathrm{m}^{2}\right]\right), \mathrm{n}(\%)$ & $2(4.4)$ \\
Obesidad (IMC $\left.\geq 30\left[\mathrm{~kg} / \mathrm{m}^{2}\right]\right), \mathrm{n}(\%)$ & $7(15.6)$ \\
Disnea 1 y $2(\mathrm{MRC}), \mathrm{n}(\%)$ & $40(86.9)$ \\
Disnea grado 3 y $4(\mathrm{MRC}), \mathrm{n}(\%)$ & $6(13.1)$ \\
Número de comorbilidades [media $\pm \mathrm{DE}]$ & $1.02 \pm 0.99$ \\
Pacientes con una sola comorbilidad, $\mathrm{n}(\%)$ & $15(32.6)$ \\
\hline
\end{tabular}

* MRC, Medical Research Council. Committee on research into chronic bronchitis: Instruction for use on the questionnaire on respiratory symptoms. Devon: WJ Holman; 1966. (Referencia 5). IMC: índice de masa corporal.

Tabla 3. Resultados de las pruebas de función respiratoria de los pacientes con cáncer pulmonar localmente avanzado (etapas IIIA y IIIB) $(n=46)$.

\begin{tabular}{|c|c|}
\hline Parámetro & Valor \\
\hline CVF $\% p$ [media $\pm \mathrm{DE}]$ & $101 \pm 18$ \\
\hline $\operatorname{VEF}_{1} \% \mathrm{p}[$ media $\pm \mathrm{DE}]$ & $92.3 \pm 22.6$ \\
\hline $\operatorname{VEF}_{1}^{1} / \mathrm{CVF}<0.7, \mathrm{n}(\%)$ & $18(39.1)$ \\
\hline $\mathrm{DLCO} \mathrm{mL} / \mathrm{min} / \mathrm{mmHg}[$ media $\pm \mathrm{DE}]$ & $18.2 \pm 7.3$ \\
\hline $\mathrm{DLCO} \% \mathrm{p}[$ media $\pm \mathrm{DE}]$ & $78.8 \pm 27$ \\
\hline Carboxihemoglobina \%, [media $\pm \mathrm{DE}]$ & $1.5 \pm 0.6$ \\
\hline $\mathrm{PaO}_{2} \mathrm{mmHg},[$ media $\pm \mathrm{DE}]$ & $62.7 \pm 7.1$ \\
\hline Hipoxemia $\left(\mathrm{PaO}_{2}<55 \mathrm{mmHg}\right), \mathrm{n}(\%)$ & $7 / 42(16.7)$ \\
\hline $\mathrm{PaO}_{2}$ limítrofe $(\geq 55 \mathrm{y}<59 \mathrm{mmHg}), \mathrm{n}(\%)$ & $6 / 42(14.3)$ \\
\hline
\end{tabular}

CVF: capacidad vital forzada; $V_{E F}$ : volumen espiratorio forzado en el primer segundo; DLCO: difusión pulmonar de monóxido de carbono; $\mathrm{PaO}_{2}$ : presión parcial de oxígeno en sangre arterial. 
Tabla 4. Calidad de sueño de los pacientes con cáncer pulmonar localmente avanzado (etapas IIIA y IIIB) $(n=46)$. Todos los datos son $\mathrm{n}(\%)$.

\begin{tabular}{|c|c|c|c|c|}
\hline \multirow[b]{2}{*}{ Características del sueño } & \multicolumn{4}{|c|}{ Pittsburgh Sleep Quality Index (PSQI)* } \\
\hline & 0 mejor calidad & 1 & 2 & 3 peor calidad \\
\hline Calidad & $19(42.2)$ & $17(37.8)$ & $8(17.8)$ & $1(2.2)$ \\
\hline Latencia & $21(46.7)$ & $16(35.6)$ & $6(13.3)$ & $2(4.4)$ \\
\hline Duración & $15(32.6)$ & 19 (41.3) & $6(13.5)$ & $6(13.5)$ \\
\hline Eficiencia & $22(47.8)$ & $9(19.6)$ & $6(13.0)$ & $9(19.6)$ \\
\hline Alteraciones del sueño & $1(2.2)$ & $36(78.3)$ & $8(17.3)$ & $1(2.2)$ \\
\hline Uso de sedantes & $40(87.0)$ & $0(0)$ & $1(2.1)$ & $5(10.9)$ \\
\hline Disfunción diurna & $18(39.1)$ & $23(50.0)$ & $4(8.7)$ & $1(2.2)$ \\
\hline
\end{tabular}

* En esta tabla mostramos la distribución de cada uno de los dominios del cuestionario de calidad de sueño de Pittsburgh, los cuales son: calidad, latencia, duración, eficiencia, alteraciones del sueño, uso de sedantes y disfunción diurna. Por ejemplo, para la pregunta "Durante el último mes, ¿cómo valoraría en conjunto, la calidad de su sueño?”, el $42 \%$ de los pacientes en el dominio de calidad refirieron tener buena calidad del sueño (puntaje de cero): 0 = muy buena, 1 = bastante buena, 2 = bastante mala, y $3=$ muy mala. El resto de los pacientes respondieron que tenían una calidad: 1 = bastante buena (37.8\%), 2 = bastante mala $(17.8 \%)$ y $3=$ muy mala $(2.2 \%)$. El total del dominio de calidad da un $100 \%$ de respuestas a esta pregunta de 45 pacientes. La media del puntaje total fue de $6.2 \pm 4.1$ (mínimo-máximo de 0-19). Fueron 23/46 (50\%) que tuvieron buena calidad del sueño (puntaje total $\leq 5)$ y $23 / 46(50 \%)$ mala calidad del sueño (puntaje total > 5). ${ }^{*}$ Buysse DJ, Reynolds CF, Monk TH, Berman SR, Kupfer DJ. The Pittsburgh Sleep Quality Index: A new instrument for psychiatric practice and research. Psychiatry Research 1989;28(2):193-213. (Referencia 6).

Tabla 5. Calidad de vida evaluada por el cuestionario EuroQol en pacientes con cáncer pulmonar localmente avanzado (etapas IIIA y IIIB) $(\mathrm{n}=46)$. Todos los datos son $\mathrm{n}(\%)^{*}$.

\begin{tabular}{lccc}
\hline Dominio & 1 (sin problemas) & 2 (algunos problemas) & 3 (problemas graves) \\
\hline Movilidad & $38(82.6)$ & $6(13.0)$ & $2(4.4)$ \\
Cuidado personal & $44(95.6)$ & $1(2.2)$ & $1(2.2)$ \\
Actividades cotidianas & $35(76.1)$ & $10(21.7)$ & $1(2.2)$ \\
Malestar y dolor & $25(54.4)$ & $18(39.1)$ & $3(6.59)$ \\
Ansiedad y depresión & $37(80.4)$ & $8(17.4)$ & $1(2.2)$ \\
\hline
\end{tabular}

* Measuring Self-Reported Population Health: An International Perspective based on EQ-5D. Edited by Agota Szende and Alan Williams On behalf of: The EuroQol Group's International Task Force on Self-Reported Health. ${ }^{\odot}$ 2004. EuroQol Group. ISBN 9639456470.

Tabla 6. Asociación entre calidad del sueño (variable dependiente: mala calidad del sueño $=$ puntaje total $\leq 5 \mathrm{y}$ buena calidad del sueño $=$ puntaje total $>$ 5) y calidad de vida. Resultados del análisis multivariado mediante regresión logística no condicional. ${ }^{*}$

\begin{tabular}{lcc}
\hline Variable & RM (IC95\%) & $\mathrm{p}$ \\
\hline $\begin{array}{l}\text { Dolor (Pittsburgh Sleep Quality } \\
\text { Index [PSQI]) }\end{array}$ & $6.5(1.1-39.4)$ & 0.04 \\
Ansiedad y depresión (EQ-5D) & $33.3(2.2-505)$ & 0.01 \\
\hline
\end{tabular}

${ }^{*}$ Controlando por malestar y dolor (EQ.5D), cuidado personal y último grado de escolaridad alcanzado.

total de los cinco dominios de la escala resultó en una media de $0.848(E E \pm 0.185)$ mientras que la mediana y los valores mínimos y máximos para el puntaje total del EQ-5D fue de 0.843 (0.086-1.0). El promedio y la mediana del puntaje revelan una buena calidad de vida en los pacientes al inicio del estudio (tabla 5). La percepción de la propia salud, otra evaluación del EuroQol (EQ VAS), fue de $77 \pm 19$ en una escala de 0 a 100

El análisis multivariado mostró que la mala calidad del sueño se asoció significativamente con la presencia de dolor (PSQI) $(\mathrm{RM}=6.5, \mathrm{IC} 95 \%$ [1.1-39.4], $\mathrm{p}=$ $0.04)$ y con ansiedad y depresión ( $E Q-5 D)(R M=33.3$, IC95\% [2.2-505], $p=0.01$ ), después de controlar por las variables malestar y dolor (EQ-5D), escolaridad y cuidado personal (tabla 6).

\section{DISCUSIÓN}

Los principales resultados de este estudio son: a) la tercera parte de los pacientes con cáncer pulmonar se presenta a tratamiento cuando menos con una enfermedad o condición asociada; al inicio del estudio todos los pacientes refirieron disnea, de los cuales el $13.1 \%$ 
presentó disnea grados 3 y 4; el 15.6\% fueron obesos; b) la prevalencia de EPOC evaluada por espirometría fue de $39.1 \%$; el $16.7 \%$ de los pacientes presentó hipoxemia; c) el $50 \%$ de los pacientes refirieron una mala calidad del sueño, principalmente en los dominios de duración del sueño y latencia; d) el puntaje total en la calidad de vida fue alta (0.85 [EE \pm 0.19$]$ ); sin embargo, cuando se analizan los dominios de forma individual $45.7 \%$ de los pacientes refirieron malestar y dolor y $19.6 \%$ síntomas de ansiedad y depresión, y e) la mala calidad del sueño se asoció positiva y significativamente con dolor (PSQI) y con ansiedad y depresión (EQ-5D).

La presencia de enfermedades asociadas y de disnea en pacientes con cáncer pulmonar se debe en parte a que el grupo de edad más afectado son los adultos mayores, pero también a que los factores de riesgo del cáncer, tabaquismo en forma primordial, favorecen la aparición de una variedad de enfermedades en diversos órganos y sistemas y quizá susceptibilidades compartidas. En nuestro grupo de pacientes, la edad media en años fue de $62( \pm 14)$ y no se observaron diferencias en la edad entre hombres y mujeres $(p=0.07)$. Las enfermedades que se reportaron más frecuentemente fueron: gastritis y úlcera (37\%), hipertensión arterial sistémica (28.3\%) y diabetes mellitus (17.4\%). La evaluación y control de estas comorbilidades puede mejorar el pronóstico de los pacientes con cáncer pulmonar antes del inicio de su tratamiento.

La prevalencia de enfermedades respiratorias se incrementa con la edad y contribuye en gran medida a la morbilidad y mortalidad. En nuestro estudio, el $13.1 \%$ de los pacientes con cáncer pulmonar presentaron disnea grados 3 y 4 . La disnea se atribuye habitualmente a múltiples etiologías tales como comorbilidades y pobre condición física. El tratamiento de la disnea es esencial para evitar la mala calidad de vida debida a síntomas respiratorios no tratados y no controlados..$^{10}$ Existen intervenciones farmacológicas o no farmacológicas que pueden ser útiles en el alivio de la disnea.

En cuanto a la evaluación de la función pulmonar en nuestro estudio, 39.1\% de los pacientes tuvieron EPOC diagnosticada por espirometría. El tratamiento apropiado de la EPOC puede mejorar la calidad de vida de los pacientes con cáncer pulmonar. Las comorbilidades asociadas a la EPOC incluyen enfermedad isquémica cardíaca, diabetes o cáncer pulmonar. Los broncodilatadores constituyen la forma principal del tratamiento, los $\beta$-2 agonistas y los agentes anticolinérgicos de larga duración se utilizan frecuentemente. ${ }^{11}$ Además de mejorar los síntomas, se considera que estos tratamientos pueden ejercer algún grado de modificación de la enfermedad. En pacientes con cáncer pulmonar, la EPOC puede ser controlada mediante tratamiento médico antes del inicio de la quimio y radioterapia. En nuestro estudio, los hombres presentaron una frecuencia significativamente mayor de EPOC que las mujeres (45.8\% vs. 13.6\%, RM = 5.4, [IC95\% 1.1-34.5], $p=0.01$ ) respectivamente (datos no mostrados), lo cual se había reportado previamente. La mayor parte de las mujeres tienen pruebas de función respiratoria normales al momento del diagnóstico de cáncer pulmonar, lo que sugiere que las diferencias en la función pulmonar observadas entre ambos sexos deben ser tomada en cuenta durante el tamizaje para detectar cáncer de pulmón, ya que basar la estratificación de alto riesgo de cáncer con la presencia de EPOC puede excluir a una proporción significativa de mujeres con cáncer pulmonar. $^{12}$

Además de la baja función pulmonar observada en pacientes con EPOC, el cáncer pulmonar también se ha visto asociado a una mayor disminución en la función pulmonar, lo que se refleja en nuestro grupo de pacientes (tabla 3). Un estudio de casos y controles confirmó las observaciones previas de que la obstrucción al flujo de aire es un factor de riesgo independiente de cáncer pulmonar. Estos investigadores encontraron que una disminución del $\mathrm{VEF}_{1}$ y del cociente $\mathrm{VEF}_{1} / \mathrm{CVF}$ estuvieron significativamente asociados a un diagnóstico de cáncer pulmonar con una RM de 1.15 (IC95\%, 1.00-1.32; $\mathrm{p}=.046)$ y una RM de 1.29 (IC95\% 1.02-1.62; $p=.031$ ), respectivamente. En este mismo estudio no se observó asociación entre la extensión del enfisema y el riesgo de cáncer pulmonar. ${ }^{13}$ Se ha destacado el papel de la rehabilitación pulmonar de los pacientes con cáncer pulmonar como estrategia para mejorar la calidad de vida de esta población. ${ }^{14}$

En otro estudio clínico, la difusión de monóxido de carbono (DLCO) en pacientes con cáncer pulmonar fue baja en comparación con un grupo control; cuando los pacientes y los controles (EPOC) se parearon por índice tabáquico y función pulmonar, la DLCO se mantuvo significativamente baja en el grupo de cáncer pulmonar (56.4 \pm 12.8 en porcentaje del predicho) comparado con el grupo control $(64.4 \pm 20.1$ en porcentaje del predicho; $p=0.0027$ ). A decir de los autores, esta reducción podría deberse a la coexistencia de enfisema y cáncer pulmonar. ${ }^{15}$

En nuestro estudio, el $16.7 \%$ de los pacientes presentaron hipoxemia con una presión parcial de oxígeno en sangre arterial < $55 \mathrm{mmHg}$; además, identificamos $14.3 \%$ de pacientes que tenían oxigenación limítrofe de 55 a $59 \mathrm{mmHg}$. De acuerdo a nuestros datos, el $31 \%$ de los pacientes con cáncer pulmonar requieren de la administración de oxígeno antes de su inicio de tratamiento. Las enfermedades respiratorias crónicas por mecanismos diversos, pueden generar insuficiencia 
respiratoria (hipoxemia); por ello, los pacientes deben recibir oxígeno suplementario como parte de su tratamiento. Se ha demostrado que en pacientes con EPOC en tratamiento con oxígeno suplementario a largo plazo, disminuyen las causas de mortalidad respiratoria. Estos resultados enfatizan la necesidad de tratar las comorbilidades asociadas a EPOC y cáncer pulmonar para reducir la mortalidad por todas las causas y por enfermedades cardiovasculares y respiratorias. ${ }^{16} \mathrm{La}$ hipoxemia nocturna es muy común en pacientes con cáncer pulmonar y puede contribuir a la dificultad para concentrarse que reportan estos pacientes. ${ }^{17}$

En nuestro estudio, el $50 \%$ de los pacientes con cáncer manifestaron mala calidad de sueño, siendo los dominios más afectados el de la eficiencia y la duración del sueño. Nuestros resultados son consistentes con los hallazgos de otros investigadores que han demostrado latencias al sueño prolongadas o un tiempo total del sueño reducido. ${ }^{18}$ Existe evidencia de que un programa de ejercicio llevado a cabo en el domicilio mejora la función, la fatiga y la calidad del sueño en pacientes con cáncer pulmonar avanzado. ${ }^{19}$ Las alteraciones del sueño originan un gran malestar en los pacientes con cáncer. Los trastornos del sueño están asociados con una mayor frecuencia de dolor, depresión, ansiedad y una peor sensación de malestar. Se ha sugerido que esos cuatro síntomas deben ser evaluados en los pacientes con cáncer avanzado que reportan alteraciones del sueño. Se ha sugerido como punto de corte ideal del ESAS-sleep ítem para tamizaje de trastornos del sueño un puntaje de $\geq 3$ (Edmonton Symptom Assessment Scale [ESAS], por sus siglas en inglés). ${ }^{20}$ Otros estudios clínicos han mostrado que los pacientes con alteraciones del estado de ánimo reportan una mayor frecuencia de alteraciones del sueño. Los autores sugieren que los pacientes oncológicos necesitan ser evaluados, tanto para bienestar mental como para trastornos del sueño. ${ }^{21}$ La privación del sueño y la mala calidad del mismo tienen un impacto negativo en la calidad de vida. El reconocimiento y corrección de la hipoxemia nocturna y otras alteraciones del sueño pueden también mejorar la calidad de vida. Los pacientes con diagnóstico incidente de cáncer pulmonar refieren marcadas alteraciones del sueño, somnolencia diurna excesiva y una pobre calidad de vida. Se sugiere el uso de tratamientos farmacológicos y no farmacológicos para mejorar la calidad de vida. En conclusión, los pacientes con cáncer pulmonar avanzado necesitan ser evaluados para identificar alteraciones del sueño antes del inicio de la radioterapia. ${ }^{22}$

Respecto a la calidad de vida, el puntaje total de los cinco dominios de la escala del EuroQol resultó en una media y desviación estándar de 0.85 ( \pm 0.19$)$, lo que refleja una buena calidad de vida; sin embargo, cuando se analizan los dominios de forma independiente el $45.7 \%$ reportaron dolor o malestar y el $19.6 \%$ reportaron signos de ansiedad y depresión grados 3 y 4 . Se ha descrito previamente que en pacientes con cáncer pulmonar, los dominios de calidad de vida predictores de supervivencia son el funcionamiento físico y el dolor. Los resultados de un estudio previo mostraron que para cada sitio de cáncer, al menos un dominio del HRQOL ofreció información pronóstica que tuvo un efecto aditivo independientemente de las variables clínicas y sociodemográficas. ${ }^{23}$ En nuestro estudio la percepción de la propia salud, otra evaluación del EuroQol (EQ VAS), presentó una media de $77 \pm 19$, puntaje que revela una pobre o regular percepción de la propia salud.

Los pacientes con cáncer pulmonar localmente avanzado experimentan una calidad de vida global comprometida, y un funcionamiento social relativamente bajo durante los primeros tres meses después del diagnóstico de cáncer. ${ }^{24}$ Las comparaciones entre el tipo y número de cánceres para los cuales un individuo recibe tratamiento, muestran peor calidad de vida en aquellos pacientes tratados por cáncer pulmonar, seguidos de aquellos que estaban en tratamiento por más de un tipo de cáncer. ${ }^{25}$ En pacientes con diagnóstico incidente de cáncer pulmonar avanzado, los individuos con menor actividad física al momento del diagnóstico mostraron una debilidad mayor y una proporción mayor de síntomas depresivos. ${ }^{26}$ En nuestro estudio $19.6 \%$ de los pacientes presentaron síntomas de ansiedad y depresión al momento del ingreso al estudio. Estos síntomas pueden ser tratados antes del inicio del tratamiento oncológico y de esta manera mejorar, posiblemente, el pronóstico de los pacientes. Un estudio de 89 pacientes con cáncer pulmonar mostró que los predictores de ideación suicida fueron la presencia de dolor y el haber desarrollado un episodio de depresión mayor, por lo que los autores sugieren que el manejo adecuado del dolor y la intervención psiquiátrica durante el tratamiento o previamente al mismo pueden mejorar el pronóstico de estos pacientes. ${ }^{27}$

Nuestro estudio tiene como limitación el escaso número de pacientes estudiados; sin embargo, fueron pacientes estrictamente caracterizados, reclutados por dos años y en quienes se realizaron métodos de clinimetría y de función pulmonar sólidamente estandarizados. En este estudio describimos las comorbilidades y condiciones médicas presentes en los pacientes con cáncer pulmonar avanzado, que pueden ser tratadas o prevenidas antes del inicio de la quimio o radioterapia. Lo anterior podría mejorar la calidad del sueño, la calidad de vida y el pronóstico de los pacientes con cáncer pulmonar. 


\section{Agradecimientos}

Este proyecto obtuvo financiamiento del CONACYT, número de proyecto: 180466.

\section{REFERENCIAS}

1. Wang JW, Gong XH, Ding N, et al. The influence of comorbid chronic diseases and physical activity on quality of life in lung cancer survivors. Support Care Cancer 2015;23(5):1383-1389. doi: 10.1007/s00520014-2494-7.

2. Aarts MJ, Aerts JG, van den Borne BE, Biesma B, Lemmens VE, Kloover JS. Comorbidity in patients with small-cell lung cancer: trends and prognostic impact. Clin Lung Cancer 2015;16(4):282-291. doi: 10.1016/ j.cllc.2014.12.003.

3. Menezes AM, Victora CG, Perez-Padilla R; PLATINO Team. The Platino project: methodology of a multicenter prevalence survey of chronic obstructive pulmonary disease in major Latin American cities. BMC Med Res Methodol 2004;4:15.

4. López-Varela MV, Montes-de Oca M, Halbert R, et al. Comorbidities and health status in individuals with and without COPD in five Latin American cities: the PLATINO study. Arch Bronconeumol 2013;49(11):468-474. doi: 10.1016/j.arbres.2013.05.003.

5. Medical Research Council. Committee on research into chronic bronchitis: Instruction for use on the questionnaire on respiratory symptoms. Devon: W J Holman; 1966.

6. Buysse DJ, Reynolds CF, Monk TH, Berman SR, Kupfer DJ. The Pittsburgh Sleep Quality Index: a new instrument for psychiatric practice and research. Psychiatry Research 1989;28(2):193-213.

7. Shaw JW, Johnson JA, Coons SJ. US valuation of the EQ-5D health states: development and testing of the $D 1$ valuation model. Med Care 2005;43(3):203220.

8. Miller MR, Hankinson J, Brusasco V, et al. Standardisation of spirometry. Eur Respir J 2005;26(2):319338.

9. Stata Technical Support. Citing Stata software, documentation, and FAQs; Date November 1999; updated June 2013.

10. Akgün KM, Crothers K, Pisani M. Epidemiology and management of common pulmonary diseases in older persons. J Gerontol A Biol Sci Med Sci 2012;67(3):276291. doi: $10.1093 /$ gerona/glr251.

11. Decramer M, Janssens W, Miravitlles M. Chronic obstructive pulmonary disease. Lancet 2012;379(9823):13411351. doi: 10.1016/S0140-6736(11)60968-9.

12. Loganathan RS, Stover DE, Shi W, Venkatraman E. Prevalence of COPD in women compared to men around the time of diagnosis of primary lung cancer. Chest 2006;129(5):1305-1312.

13. Maldonado F, Bartholmai BJ, Swensen SJ, Midthun DE, Decker PA, Jett JR. Are airflow obstruction and radio- graphic evidence of emphysema risk factors for lung cancer? A nested case-control study using quantitative emphysema analysis. Chest 2010;138(6):1295-1302. doi: 10.1378/chest.09-2567.

14. Rivas-Perez $\mathrm{H}$, Nana-Sinkam P. Integrating pulmonary rehabilitation into the multidisciplinary management of lung cancer: A review. Respir Med 2015;109(4):437-442. doi:10.1016/j.rmed.2015.01.001.

15. Barreto SS, McClean PA, Szalai JP, Zamel N. Reduction of lung diffusion for carbon monoxide in patients with lung carcinoma. Chest 1993;103(4):11421146.

16. Ekström MP, Wagner P, Ström KE. Trends in causespecific mortality in oxygen-dependent chronic obstructive pulmonary disease. Am J Respir Crit Care Med 2011;183(8):1032-1036. doi: 10.1164/rccm.201010$17040 \mathrm{C}$

17. Wilcock A, England R, El Khoury B, et al. The prevalence of nocturnal hypoxemia in advanced cancer. J Pain Symptom Manage 2008;36(4):351-357. doi: 10.1016/j. jpainsymman.2007.11.007.

18. Dean GE, Redeker NS, Wang YJ, et al. Sleep, mood, and quality of life in patients receiving treatment for lung cancer. Oncol Nurs Forum 2013;40(5):441-451. doi: 10.1188/13.ONF.441-451.

19. Cheville AL, Kollasch J, Vandenberg J, et al. A homebased exercise program to improve function, fatigue, and sleep quality in patients with Stage IV lung and colorectal cancer: a randomized controlled trial. J Pain Symptom Manage 2013;45(5):811-821. doi: 10.1016/j. jpainsymman.2012.05.006.

20. Delgado-Guay M, Yennurajalingam S, Parsons H, Palmer JL, Bruera E. Association between selfreported sleep disturbance and other symptoms in patients with advanced cancer. J Pain Symptom Manage 2011;41(5):819-827. doi: 10.1016/j.jpainsymman.2010.07.015.

21. van Onselen C, Dunn LB, Lee K, et al. Relationship between mood disturbance and sleep quality in oncology outpatients at the initiation of radiation therapy. Eur J Oncol Nurs 2010;14(5):373-379. doi: 10.1016/j. ejon.2009.12.002.

22. Dean GE, Abu-Sabbah E, Yingrengreung S, et al. Sleeping with the enemy: sleep and quality of life in patients with lung cancer. Cancer Nurs 2015;38(1):60-70. doi: 10.1097/NCC.0000000000000128.

23. Quinten C, Martinelli F, Coens C, et al; Patient Reported Outcomes and Behavioral Evidence (PROBE) and the European Organization for Research and Treatment of Cancer (EORTC) Clinical Groups. A global analysis of multitrial data investigating quality of life and symptoms as prognostic factors for survival in different tumor sites. Cancer 2014;120(2):302-311. doi: 10.1002/ cncr. 28382.

24. Liao YC, Shun SC, Liao WY, Yu CJ, Yang PC, Lai YH. Quality of life and related factors in patients with newly diagnosed advanced lung cancer: a longitudinal study. Oncol Nurs Forum 2014;41(2):E44-55. doi: 10.1188/14. ONF.E44-E55. 
25. Baker F, Haffer SC, Denniston M. Health-related quality of life of cancer and non cancer patients in Medicare managed care. Cancer 2003;97(3):674-681.

26. Granger $\mathrm{CL}$, McDonald $\mathrm{CF}$, Irving $\mathrm{L}$, et al. Low physical activity levels and functional decline in individuals with lung cancer. Lung Cancer 2014;83(2):292-299. doi: 10.1016/j.lungcan.2013.11.014.

27. Akechi T, Okamura $\mathrm{H}$, Nishiwaki Y, Uchitomi Y. Predictive factors for suicidal ideation in patients with unresectable lung carcinoma. Cancer 2002;95(5):1085-1093. $\triangle$ Correspondencia:

Dra. en C. Cecilia García-Sancho

Instituto Nacional de Enfermedades Respiratorias Ismael Cosío Villegas. Calzada de Tlalpan Núm. 4502, Colonia Sección XVI, 14080, México, D.F. Teléfono: 54-87-17-00, extensión 5238, fax: 56-65-46-23

Correo electrónico: cegarsan@netscape.net; cegarsanfi@gmail.com

Los autores declaran no tener conflicto de intereses. 